\title{
MRS Elects Officers, Councillors for 1998
}

Members of the Materials Research Society have elected two officers and six councillors to join the 1998 Council, which consists of the executive committee and 15 councillors. The annual election ended October 10, 1997.

\section{MRS Officers}

President

Robert J. Nemanich (1998)

North Carolina State University

Immediate Past President

Robert Hull (1998)

University of Virginia

Vice President (President-Elect)

*Ronald Gibala (1998)

University of Michigan

Secretary: *Chuang Chuang Tsai (1999)

Applied Komatsu Technology

Treasurer: Alan J. Hurd (1998)

Sandia National Laboratories
1998 MRS Councillors

Cammy R. Abernathy (1998)

University of Florida

Harry A. Atwater (1998)

California Institute of Technology

*Michael J. Aziz (2000)

Harvard University

*Katayun Barmak (2000)

Lehigh University

Theodore M. Besmann (1999)

Oak Ridge National Laboratory

*David J. Eaglesham (2000)

Bell Laboratories, Lucent Technologies

Martin L. Green (1998)

Bell Laboratories, Lucent Technologies

*Alexander H. King (2000)

State University of NY at Stony Brook
*Karen Maex (2000)

IMEC, Belgium

Amy J. Moll (1999)

Hewlett Packard

Virginia M. Oversby (1998)

VMO Konsult

Tim Sands (1999)

University of California-Berkeley

Lyle H. Schwartz (1998)

Associated Universities, Inc.

*Alan I. Taub (2000)

Ford Motor Company

James S. Williams (1998)

Australian National University

(Terms of office expire at the end of the years indicated in parentheses.)

*Newly elected.

\section{Graduate Student Award Finalists Compete at 1997 MRS Fall Meeting}

Twenty-six finalists will compete for the MRS Graduate Student Awards to be presented during the awards ceremony on Wednesday, December 3, at 6:00 p.m., in the Boston Marriott Hotel, Salon E at the 1997 MRS Fall Meeting. The Graduate Student Award Special Talk Session, in which each finalist gives a 10-minute presentation, will be held on Monday, December 1, at noon.

Following is the list of finalists, their places of study, titles of papers, and the symposium or symposia in which each finalist is participating:

Kathleen M. Amm, Florida State University, "Processing and Properties of $(\mathrm{Hg}, \mathrm{Bi}) \mathrm{Ba}_{2} \mathrm{Ca}_{2} \mathrm{Cu}_{3} \mathrm{O}_{y}$ Tapes" and "Synthesis and Processing of Bi-Doped $\mathrm{Hg}$-1223 Superconductor" (Symposium T)

Martin Z. Bazant, Harvard University, "Environment-Dependent Interatomic Potential for Bulk Silicon" (Symposium R)

J. D. Brown, North Carolina State University, "The First Nitride Laser Diode on Silicon Carbide" (Symposium D)

Lila J. Chamberlain, Massachusetts Institute of Technology, "Cellular Reaction to Synthetic and Natural Polymeric Tube Implants Used for Peripheral Nerve Regeneration" (Symposium O)

Danielle R. Chamberlin, University of CaliforniaBerkeley, "Multivalent Acceptor Doped Germanium Lasers: A Solid-State Tunable Source From 75 to $300 \mathrm{~mm}^{\prime \prime}$ (Symposium F)

Jinghong Chen, University of Southern California, "High E-O Coefficient Polymers Based on a Chromophore Containing Isophorone Moiety for Second-Order Nonlinear Optics" (Symposium J)

Anthony D. Dinsmore, University of Pennsylvania, "Hard Spheres Inside Vesicles: Depletion Forces and Membrane Curvature" (Symposium L)

Stephen A. Empedocles, Massachusetts Institute of Technology, "Photoluminescence Spectroscopy of Single CdSe Nanocrystallite Quantum Dots"

\section{(Symposium C)}

Michael Fasolka, Massachusetts Institute of Technology, "Observed Substrate TopographyMediated Lateral Patterning of Diblock Copolymer Films" (Symposium N)

Jennifer A. Hollingsworth, Washington University, "Spray Chemical Vapor Deposition of $\mathrm{CuInS}_{2}$ Thin Films: The Influence of Deposition Conditions on Film Quality" and "LowTemperature, Solution-Based Route to NanoIndium Sulfide Phases and a New Chemical-Bath Process for Deposition of Orthorhombic InS Thin Films" (Symposium W)

C. Esther Jesurum, Massachusetts Institute of Technology, "Modeling Collision Cascade Structure of $\mathrm{SiO}_{2}, \mathrm{Si}_{3} \mathrm{~N}_{4}$, and $\mathrm{SiC}$ Using Local Topological Approaches" (Symposium KK)

Bijaya B. Karki, University of Edinburgh, "High Pressure Elasticity of the Major Silicate and Oxide Minerals of the Earth's Lower Mantle" (Symposium DD)

Ilya Koltover, University of California-Santa Barbara, "Structure and Interactions in SelfAssembled DNA-Cationic Lipid Complexes in the Presence of Single and Multivalent Counterions" and "Melting and Interactions in Multilayers of Two-Dimensional Crystals of Membrane-Protein Bacteriorhodopsin" (Symposia $K$ and $L$ )

Francois Leonard, University of Toronto, "Alloy Decomposition and Surface Instabilities in Thin Films" and "Phase Separation in Heteroepitaxial Thin Film Growth" (Symposium A)

Alexander V. Mamishev, Massachusetts Institute of Technology, "Measurement of Stratified Distributions of Dielectric Properties and Dependent Physical Variables" (Symposium EE)

Jeffery B. Maxson, University of WisconsinMadison, "Comparative Studies of the Surface of GaN (0001) Thin Films with LEEM" (Symposium D)
Robert C. Mucic, Northwestern University, "Functional DNA/Nanoparticle-Based Materials" (Symposium FF)

Michael Natusch, University of Cambridge, "Local Electronic Structure of Defects in GaN from Spatially Resolved Electron Energy-Loss Spectroscopy (EELS)" (Symposium D)

Ainissa G. Ramirez, Stanford University, "Influence of Annealing on the Microstructure of Amorphous Carbon Thin Films for Magnetic Hard Disks" (Symposium AA)

Rajesh A. Rao, Duke University, "Effect of Substrate Miscut and Lattice Mismatch on Grown Mechanisms of Epitaxial Metallic Oxide $\mathrm{SrRuO}_{3}$ Thin Films" and "Effect of Domain Structure on the Magnetoresistance of Epitaxial Thin Films of Ferromagnetic Metallic Oxide $\mathrm{SrRuO}_{3}$ " (Symposia $A$ and $V$ )

Lars Rebohle, Forschungszentrum Rossendorf, "Strong Blue and Violet Light Emission from Silicon and Germanium Implanted Silicon Dioxide" (Symposium $\mathrm{H}$ )

Serge Santos, Swiss Federal Institute of Technology, "A Novel Parallel-Rotation Algorithm for Atomistic Monte Carlo Simulation of Polymer Melts and Glasses" (Symposium P)

Michael C. Wanke, University of CaliforniaSanta Barbara, "Laser CVD Rapid Processing of Ceramic Photonic Band-Gap Microstructures" (Symposium W)

Andre Wong, University of British Columbia "Stability of Magnesium Implanted $\mathrm{YBa}_{2} \mathrm{Cu}_{3} \mathrm{O}_{7}$ Thin Films" (Symposium T)

Ke Yang, University of Massachusetts, "Three Dimensional Quantum Confinement in Gallium Nitride Nanoparticles" and "Characterizing the NLO Chromophore Orientation of Polymeric Film by Electroabsorption Spectroscopy" (Symposia C and J)

Stefano Zapperi, Boston University, "Driven Dynamics of a Ferromagnetic Domain Wall: A Theory for the Barkhausen Effect" (Symposium B) 
W1⿴囗十

FALL' 97

Complimentary Reception will be held in University Hall on Tuesday evening from 5:00 p.m. to 6:30 p.m.

\section{BOSTON MARRIOTT HOTEL AND WESTIN HOTEL/COPLEY PLACE}

The MRS Exhibit, held in conjunction with the 1997 MRS Fall Meeting, will encompass the full spectrum of equipment, instrumentation, products, software, publications and services for materials research. As always, the exhibit will closely parallel the nature of the symposia, and the technical program has been arranged to allow meeting participants ample opportunity to attend the exhibit. MRS encourages attendees to visit the exhibit by scheduling coffee breaks, deli-style lunches, and a meeting-wide reception in University Hall.

\begin{tabular}{|lcc|}
\multicolumn{1}{c|}{ EXHIBIT HOURS } \\
\hline \multicolumn{1}{|c|}{$\begin{array}{c}\text { Marriott Hotel } \\
\text { University Hall \& Atrium Lounge }\end{array}$} & $\begin{array}{c}\text { Westin Hotel } \\
\text { 3rd \& 4th Floors }\end{array}$ \\
\hline Tuesday, December 2 & $11: 30$ a.m. - 6:30 p.m. & $9: 30$ a.m. - 5:00 p.m. \\
Wednesday, December 3 & $9: 30$ a.m. - 5:00 p.m. & $9: 30$ a.m. - 5:00 p.m. \\
& & $7: 30$ p.m. - 10:00 p.m. \\
Thursday, December 4 & $9: 30$ a.m. - 2:30 p.m. & $9: 30$ a.m. - 1:30 p.m. \\
\hline
\end{tabular}

\section{PARTIAL LIST OF 1997 FALL EXHIBITORS}

\section{Products \& Services}

A \& N Corporation

Advanced Research Systems

AIXTRON, Inc.

AJA International, Inc

Alcatel Vacuum Products, Inc.

Aldrich Chemical Company, Inc.

Alfa Aesar, a Johnson Matthey Company

Amptek, Inc.

Andeen-Hagerling, Inc.

Angstrom Sciences

APD Cryogenics, Inc.

ASTeX/Applied Science and

Technology, Inc.

Australian Scientific Instruments

Barnstead/Thermolyne Corporation

Barr Associates, Inc.

Bede Scientific Incorporated

BetaBeam

Bio-Logic Co.

Blake Industries, Inc.

Bruker Analytical X-Ray Systems, Inc.

Buehler Ltd.

Burleigh Instruments, Inc.

Cameca Instruments, Inc.

CAPE Simulations, Inc.

CERAC, Inc.

Ceramaseal

CHA Industries

Chemglass, Inc.

Commonwealth Scientific

Corporation

Cree Research, Inc.

CRI, Inc.

Cryomech, Inc.

CrysTec

DCA Instruments, Inc.

Denton Vacuum, Inc.

Digital Instruments, Inc.

Diversified Technologies, Inc.

Duniway Stockroom Corp.

Dynamic Systems Inc.

EDAX International

Edwards High Vacuum International

EMCORE Corporation

EMiSPEC Systems, Inc.

Epichem Inc.

EPI MBE Products Group

Epion/PVD Products

Epitaxial Technologies, LLC

Epitronics Corp./ATMI

ESCETE B.V.

ESM Software

Evans East
E.A. Fischione Instruments, Inc.

Gatan, Inc.

General Vacuum, Inc.

Glassman High Voltage, Inc.

Goodfellow Corporation

High Voltage Engineering

Europa B.V.

Hinds Instruments, Inc.

Hitachi Scientific Instruments

Huntington Mechanical

Laboratories, Inc.

Hysitron Incorporated

IBM Analytical and Test Services

Implant Sciences Corporation

Industrial Science \& Technology Network

Inel, Inc.

Innotec Group, Inc.

InnoVac Corporation

Innovative Technology, Inc.

Inorgtech

Instruments SA, Inc.

Insulator Seal Incorporated

Ion Tech, Inc.

Janis Research Company, Inc.

JCPDS-ICDD

JEOL USA, Inc.

Johnson Matthey Electronics

k-Space Associates, Inc.

Kaiser Optical Systems, Inc.

Keithley Instruments, Inc.

Kevex Instruments, Inc.

Kimbal Physics Inc.

KLA-Tencor

Kratos Analytical Inc.

Ladd Research Industries, Inc.

Lake Shore Cryotronics, Inc.

Lambda Physik, Inc.

Lambda Technologies

Lehighton Electronics, Inc.

Kurt J. Lesker Company

Leybold Inficon Inc.

LUXTRON Corporation

Magnet Sales \& Manufacturing

MARCH Instruments, Inc.

M. Braun, Inc.

MCNC

MDC Vacuum Products Corporation

Micrion Corporation

Micro Photonics, Inc.

Microcal Software, Inc.

Micropyretics Heaters International Inc. (MHI Inc.)

MKS Instruments, Inc.

MMR Technologies, Inc

Modasco, Inc.
Molecular Metrology, Inc.

Molecular Simulations, Inc.

Morton International, Inc.

MTI Corporation

Nano Instruments, Inc.

National Electrostatics Corporation

Naval Research Laboratory (NRL)

Neocera, Inc.

NFT-Nanofilm Technologie GmbH

Nicolet Instrument Corporation

NORAN Instruments, Inc.

Noranda Advanced Materials

NOVOCONTROL

NYS Center for Advanced Thin

Film Technology

Olympus America Inc

Omicron Associates

On-Line Technologies, Inc.

Osaka Vacuum, Ltd.

Osmic, Inc.

Oxford Applied Research

Oxford Instruments America, Inc.

Park Scientific Instruments

Pfeiffer Vacuum Technology

Philips Analytical X-Ray

Philips Electron Optics Inc.

Physical Electronics, Inc.

Praxair Specialty Ceramics

Princeton Gamma-Tech, Inc.

Princeton Instruments, Inc.

Princeton Scientific Corp.

Pure Tech, Inc.

Quad Group, Inc.

Quantum Design, Inc.

Quesant Instrument Corporation

Radiant Technologies, Inc.

Renishaw Inc.

Research and PVD Materials

Corporation

Riber Division/ISA Inc.

Rigaku/USA, Inc.

RJ Lee Instruments, Ltd.

RMC

SC Technology/Telemark

Scintag Inc.

SiCrystal AG

SKION Corporation

Solartron Inc

Soleras Ltd.

SOPRA, Inc.

South Bay Technology, Inc.

SPECS USA, Inc.

SPI Supplies/Structure Probe, Inc.

Staib Instruments

Strem Chemicals, Inc.
Structured Materials/

Nanopowder Enterprises

Struers/Logitech Product Group

Superconductive Components, Inc.

SURFACE

Surface/Interface, Inc

SurForce Corporation

SVT Associates, Inc.

Sycon Instruments, Inc.

TECHNOTRADE International, Inc.

TexSEM Laboratories, Inc. (TSL)

Thermionics Vacuum Products

Thomas Swan \& Co., Ltd.

TopoMetrix Corp.

ULTRA TEC Mfg., Inc.

Union Carbide Crystal Products

Vacuum Atmospheres Company

Vacuum Research Corporation

Varian Vacuum Products

VAT, Inc.

Virginia Semiconductor, Inc.

Voltaix, Inc.

VSI Vacuum Science

Instruments, Inc.

R.D. Webb Company

Well Diamond Wire Saws, Inc.

J.A. Woollam Co., Inc.

$X$-ray Instrumentation Associates

X-Ray Optical Systems, Inc.

Zygo Corporation

\section{Books \& Software}

Academic Press, Inc.

American Ceramic Society (ACerS)

American Chemical Society

American Institute of Physics, Inc.

American Physical Society

ASM International

Cambridge University Press

Chapman \& Hall

Compound Semiconductor

$$
\text { Magazine }
$$

Elsevier Science

Gordon and Breach/Harwood

Academic

IOP Publishing Inc

Kluwer Academic Publishers

Oxford University Press

Plenum Publishing Corporation

Springer-Verlag New York, Inc.

Taylor \& Francis

WCB/McGraw-Hill

John Wiley \& Sons, Inc. 
(as of September 1997)

ABB Extrel

Advanced Control Systems Corporation

Advanced Energy Industries, Inc. Advanced Micro Devices, Inc.

AEA Technology

Aerospace Corporation

Aetrium, Inc.

AG Associates

Air Products and Chemicals, Inc.

AIXTRON, Inc.

AKZO Nobel Chemicals, Inc.

Aldrich Chemical Company, inc.

AlliedSignal, Inc.

Aluminum Company of America

Ames Laboratory

Amptek, Inc.

AMRAY, Inc.

APD Cryogenics, Inc

Applied Materials-Applied

Komatsu Technology

Applied Materials-Implant Division

Applied Materials-RTP Product Division

ARCO Chemical Company

Argonne National Laboratory

Asahi Glass Co., Ltd.

Ashland Chemical Co.

ASM

AST elektronik GmbH

ASTeX/Applied Science and

Technology, Inc.

Baikowski International Corporation

Battelle Pacific Northwest Labs

Bede Scientific Incorporated

Bell Laboratories, Lucent

Technologies

BIOMET Incorporated

Blake Industries, Inc.

BP International Limited

Brookhaven Instruments Incorporated

Brookhaven National Laboratory

Bruker Analytical X-Ray

Systems, Inc.

Bruker Instruments, Inc.

Bunkoh-Keiki Co., Ltd.

Burleigh Instruments, Inc.

Cameca Instruments, Inc.

Canon, Inc.

CEA-LETI

Chemat Technology, Inc

Chemipro Kasei Kaisha, Ltd.

Chichibu Onoda Cement Co.

City Technology Limited

CNR-IMETEM

Coherent, Inc./Laser Group

Commonwealth Scientific Corporation

Conductus, Inc.

Consortium für Elektrochemische Industrie $\mathrm{GmbH}$

Continental Electronics Corporation

Co.Ri.M.Me

Corning Incorporated

Cree Research, Inc.

Criterion Catalyst Company L.P.

CVC

Cymer, Inc.

Dainippon Screen Manufacturing Co. Ltd.

Danfysik AVS

DCA Instruments, Inc.

Denton Vacuum, Inc.

Diamond Semiconductor Group

Diatome U.S.

Digital Instruments, Inc.

Digital Semiconductor (A Digital Equipment Corporation)
Dow Chemical Company Dow Corning Corporation dpiX, a Xerox Company

DSM Research

Dupont Company, Central

Research and Development

Dytech Corporation, Ltd.

Eastman Kodak Company

Eaton Corporation

Eaton Thermal Processing Systems

EDAX Internationa

EG\&G Instruments, Inc.

EG\&G Ortec

Electric Power Research Institute

Elsevier Science B.V.

Elsevier Science Ltd.

EMCORE Corporation

Energy Conversion Devices, Inc.

E.O. Lawrence Berkeley National Laboratory

EPI MBE Products Group

ESM Software

Charles Evans \& Associates

Evans East

Exxon Production Research Company

Exxon Research and Engineering Company

FEl Company

FHR Anlagenbau GmbH

E.A. Fischione Instruments, Inc.

Ford Motor Company

Forschungszentrum

Rossendorf e.V.

Freund Publishing House, Ltd.

FSI International

Fuji Electric Co., Ltd.

Fujikin of America, Inc

Fujikin Incorporated

Fujitsu, Ltd.

Furukawa Electric Co., Ltd.

Futaba Corp.

Gatan, Inc.

Gelest, Inc.

Genentech, Inc.

General Electric Company

General Motors Research \&

Development

GMW Associates

Goodfellow Corporation

Gordon and Breach

Gould Electronics Inc.

POWERDEX Division

Granville-Phillips Company

Harris Diamond Corporation

Hewlett Packard Company

Optoelectronics Division

Hewlett Packard Laboratories

Japan, Inc.

Hewlett Packard, NMD

High Voltage Engineering

Europa B.V.

Hitachi Europe Ltd.

Hitachi, Ltd.

Hitachi Scientific Instruments

Hoechst Celanese Corporation

Advanced Technology Grou

Hoechst Celanese Corporation

Separations Products Division

Hughes Research Laboratories

Huntington Mechanical

Laboratories, Inc.

Hysitron, Inc.

IBM Analytical and Test Services

BM Corporation-Thomas J.

Watson Research Center

$\mathrm{ICl}$ Acrylics

IMES Co., Ltd.

Initiative Scientific Products

Inorgtech, Ltd.
Institut für Schicht und

lonentechnik (ISI)

Institute for Scientific Information

Instron Corporation

Insulator Seal, Inc.

Intel Corporation

Ion Tech, Inc

ITAC, Ltd.

CPDS-International Centre for

Diffraction Data

JEOL USA, Inc.

Johnsen Ultravac

Johnson Controls, Inc.

Johnson \& Johnson Profession

Johnson Matthey Electronics

k-Space Associates, Inc.

Kaneka Corporation

Keithley Instruments, Inc.

Kimball Physics, Inc.

KLA-Tencor

Kobe Steel USA, Inc

Electronic Materials Center

Komag, Inc.

Komatsu Electronic Metals

Co., Ltd.

Kratos Analytical, Inc.

Lake Shore Cryotronics, Inc.

Lambda Physik, Inc.

Lambda Technologies, Inc.

Lawrence Livermore National

Laboratory

Leica Inc.

LEO Electron Microscopy Inc.

Kurt J. Lesker Company

Leybold Systems GmbH

Leybold Vacuum Products, Inc.

Los Alamos National Laboratory

Materials Analysis Group

Philips Semiconductors

Materials Research Corporation

Materials Research Group, Inc.

Matsushita Electric Industrial Co.

Matsushita Electronics Corp.

Mattson Technology, Inc.

MDC Vacuum Products

Corporation

MEL Chemicals

MEMC Electronic Materials, Inc.

Micrion Corporation

Micro Photonics, Inc

Microwave Research Center

Millipore Corporation

Mitsubishi Electric

Semiconductor Laboratories

Mitsubishi Materials Corporation

Mitsubishi Silicon America

Mitsui-Toatsu Chemicals, Inc.

MKS Instruments, Inc.

MMR Technologies, Inc.

Mobil Technoiogy Company

Molecular Imaging Corporation

Molecular Simulations, Inc.

Morita Chemical Industries

Morton International, Inc.

Motorola

MR Semicon, Inc.

MVSystems, Inc.

n\&k Technology, Inc

Nanophase Technologies

Corporation

NAPS France

National Electrostatics Corp.

National Semiconductor

Corporation

National Technology Transfer Center

NEC Corporation

NEC Research Institute, Inc.

Neocera, Inc.

New Focus, Inc.

Nichia Chemical Industries, Ltd.

Niki Glass Co L Ltd.

Nikko Hitech International, Inc.

Steel Corporation

Noranda Advanced Materials

Nor-Cal Products, Inc.

Northrop Grumman

Science \& Technology Cente

NSA Nissei Sangyoi America

Oak Ridge National Laboratory

Oldham S.A. France

Omicron Associates

OnTrak Systems, Inc.

Osmic, Inc.

Oxford Cryosystems

Oxford Instruments America, Inc.

Park Scientific Instruments

Parke Mathematical Laboratories

Pfeiffer Vacuum Technology, Inc.

Philips Electron Optics

Philips Electronic Instruments Company

Philips Research

Physical Electronics, Inc. (PH)

PIOLAX Incorporated

Plasma-Therm, Inc.

Plenum Publishing Corporation

Portland Cement Association

Power Reactor \& Nuclear Fue

Development Corporation

President Enterprises Co.

Princeton Gamma-Tech, Inc.

Princeton Measurements Corporation

Pure Tech, Inc.

Quantum Design, Inc.

Renishaw PLC

Research and PVD Materials Corporation

RHK Technology, Inc.

Riber Division/ISA Inc.

Rigaku/USA, Inc.

RIMCOF (R\&D Institute of Metals \& Composites for Future Industries) 